\title{
Carnets
}

Revue électronique d'études françaises de l'APEF

Deuxième série - $11 \mid 2017$

Les écrivains écrivent l'Europe en français

\section{De 1984 à 2084. Mutations de la peur totalitaire dans la dystopie européenne}

\section{Regis-Pierre Fieu}

\section{(2) OpenEdition}

Journals

\section{Édition électronique}

URL : http://journals.openedition.org/carnets/2344

DOI : 10.4000/carnets.2344

ISSN : 1646-7698

Éditeur

APEF

\section{Référence électronique}

Regis-Pierre Fieu, «De 1984 à 2084. Mutations de la peur totalitaire dans la dystopie européenne », Carnets [En ligne], Deuxième série - 11 | 2017, mis en ligne le 30 novembre 2017, consulté le 19 avril 2019. URL : http://journals.openedition.org/carnets/2344; DOI : 10.4000/carnets.2344

Ce document a été généré automatiquement le 19 avril 2019

Licence Creative Commons

Carnets est mis à disposition selon les termes de la licence Creative Commons - Atribution - Pas

d'utilisation commerciale 4.0 International. 


\title{
De 1984 à 2084. Mutations de la peur totalitaire dans la dystopie européenne
}

\author{
Regis-Pierre Fieu
}

\section{Autres temps, autres peurs}

1 À la veille de sa mort, sur son lit d'hôpital, Georges Orwell ne savait certainement pas à quel point le monde cauchemardesque qu'il avait décrit dans 1984 allait en partie se réaliser. Son intuition quant à l'essor des technologies et leur utilisation au service de la surveillance a été, à bien des égards, prophétique. Son regard sur les dictatures de son temps, précis et finalement terriblement réaliste. Ce qu'il savait certainement encore moins, c'est l'impact qu'allait avoir son œuvre sur les générations futures d'écrivains, et à quel point le modèle de son roman allait donner naissance à de nombreuses autres dystopies. Parmi ses héritiers, Boualem Sansal s'assume totalement, avec 2084, dont le seul titre est un clin d'œil au célèbre ouvrage d'Orwell. Référence assumée donc, car Sansal ne cherche pas seulement à porter l'héritage d'Orwell, mais bien à créer le 1984 de son époque, en imaginant un futur potentiel, et avertir son époque, comme le précise Richard A. Slaughter en parlant de la science-fiction :

Overall, there are two basic motivations for looking ahead and studying the future. One is to avoid dangers. The uses of foresight for this purpose are as old as humankind. The second is to set goals, dream dreams, create visions, make designs; in short, to project upon the future a wide range of purposes and intentions. (Richard A. Slaughter, 2003:2)

2 Si le monde d'Orwell était révélateur de son siècle, celui de Sansal l'est aussi. Dans le premier cas, le monde d'Oceania n'est finalement qu'une extension de l'URSS qui aurait acquis davantage de technologie et réussi à étendre le contrôle d'un parti unique, dominé par la figure de Big Brother, désormais bien connue dans la culture populaire. 1984 résumait les peurs d'une époque, celles de la dictature ultime, de la disparition des individus, de la guerre éternelle. Le spectre d'un $\mathrm{XX}^{\mathrm{e}}$ siècle ravagé par les conflits et les 
utopies devenues bien vite des dystopies. 2084 est alors, à l'instar de son aîné, un roman dystopique qui résume les peurs du $\mathrm{XXI}^{\mathrm{e}}$ siècle, que nous analyserons en détail dans cet article. 2084 pourrait apparaitre comme un simple clin d'œil, mais nous montrerons qu'il s'agit de bien plus que cela. En pastichant Orwell, Sansal veut créer le 1984 du XXI ${ }^{\mathrm{e}}$ siècle. Et en semant des détails troublants, il cherche surtout à nous expliquer que l'univers dystopique de son roman est le même que celui de 1984, dans un futur trouble. Mon article visera à illustrer cette idée, et montrer que Sansal avertit notre époque sur l'étape suivante de notre monde semi-orwellien. Cette mutation de la dystopie européenne, de la dictature politique à la dictature islamiste, indique également une peur essentielle à comprendre dans notre temps: celle qu'ont les peuples européens de voir leur terre disparaitre, leur histoire effacée, et le continent entrer dans une ère de déclin, abandonnant ses valeurs et sa culture. Cet abandon se ferait au profit d'un conquérant entré dans l'imaginaire collectif, Daech bien sûr, qui n'est sous la plume de Sansal, que l'étape primaire avant l'avènement de son monde à venir, celui, terrifiant d'Abistan, où le religieux a remplacé le politique. Nous ne pouvons prétendre, en un article, étudier tous les échos de 2084 à 1984 - la seule question de l'Histoire nécessiterait une étude approfondie - donc nous nous concentrerons sur quelques aspects, notamment les transformations qu'impliquent le changement du régime politique au régime religieux, l'aspect géographique et architectural du territoire ainsi que la place du langage. Enfin nous discuterons de la portée idéologique du roman de Boualem Sansal et notamment de sa volonté d'avertir d'un danger potentiel.

\section{Un monde religieux}

3 Le monde d'Abistan est, à l'instar d'Océania, un monde terne et obscur, dans lequel nulle joie ne semble pouvoir éclater au grand jour, où la guerre est, comme dans 1984, perpétuelle: «Le pays vivait des guerres récurrentes, spontanées et mystérieuses, cela était sûr, l'ennemi était partout, il pouvait surgir de l'est ou de l'ouest, tout autant que du nord ou du sud.» (Sansal, 2015 : 18). Dans ce climat sombre, Ati est le personnage principal. Un habitant religieux, comme les autres, habitué à suivre les commandements d'Abi, le Prophète, et surtout habitué à vivre sous l'œil implacable de Yölah, le Dieu Unique, dont la phonétique rappelle Allah, les paroles de paix en moins, la terreur en plus. 2084 commence dans l'approche de la mort, car Ati, malade, est allé faire un pèlerinage pour soigner son mal. Autour de lui s'amoncellent les cadavres et les miséreux. De sa guérison corporelle viendra sa guérison spirituelle et, plongé dans ses pensées durant plusieurs jours, Ati se rendra compte que l'univers dans lequel il vit est certainement bien plus vil qu'il ne le pense, s'il n'est tout simplement absurde. Cette conviction profonde de Sansal en la rationalité humaine est discutable, surtout dans un monde qu'il décrit lui-même comme fermé et extrêmement contrôlé, et dans lequel il apparait difficile que de telles pensées émergent, surtout ex nihilo, ce qui n'était pas le cas de Winston dans 1984, qui raisonnait à partir de sa lecture d'un livre contestataire.

4 C'est que le monde de 2084 a délaissé l'Histoire pour le Mythe, et que toutes ses fondations reposent sur l'histoire d'Abi, messager de Yölah, celui que le peuple doit écouter et suivre. Cette histoire correspond à l'idéal de société voulu par le régime: simplicité, pauvreté, obéissance. En Abistan, il est bon d'être humble, de suivre les consignes et surtout d'être un fervent croyant. La vie d'un habitant est partagée entre les prières, les pèlerinages obligatoires, les aumônes, et les dénonciations, recommandées par le régime : « Il savait 
comme les gens étaient entraînés à dénoncer, lui-même s'y appliquait avec ferveur dans son travail, son quartier, contre ses voisins et ses amis les plus sûrs.» (idem : 53) Lorsqu'elles aboutissent, les exécutions s'enchaînent, avec le regard du public, dans une arène géante où les foules en délire exultent devant la violence et se rappellent leur propre finitude devant Yölah :

Pour les fautes graves, il rejoignait les durs qui exigeaient des exécutions spectaculaires, estimant que le peuple avait droit à ces moments d'intense communion, par le sang fumant giclant à flots et la terreur purificatrice qui explosait comme un volcan. (idem : 50)

Des rituels qui rappellent 1984, notamment « the Two Minutes Hate », ces deux minutes quotidiennes consacrées à hurler sa haine face à Emmanuel Goldstein, l'Ennemi désigné du Parti. ${ }^{1}$ Le fait est que, dans 2084, cette haine n'est plus seulement tournée vers un seul ennemi, mais entre chaque personne habitant l'Abistan, indicateur fort d'un délitement du monde où la peur est omniprésente. Pourtant, l'ennemi unique existe, même si le mot a été supprimé de ce monde, comme le décrit Sansal :

L'Ennemi prit une dimension fabuleuse et épouvantable. Et un jour, sans qu'aucun signal ne fût donné, le mot Ennemi disparut du lexique. Avoir des ennemis est un constat de faiblesse, la victoire est totale ou n'est pas. On parlait de la Grande Mécréance, on parlait de makoufs, mot nouveau signifiant renégats invisibles et omniprésents. L'ennemi intérieur avait remplacé l'ennemi extérieur, ou l'inverse (... ). Lors des grandes cérémonies, on évoquait un nom chargé de toutes les peurs, le Chitan. (idem : 18)

Le Chitan est un dérivé clair de Sheitan qui signifie diable en arabe. Seulement le diable est dans 2084 l'opposant politique, l'incroyant, le renégat. Dans le monde extrêmement religieux de l'Abistan, le vocabulaire mystique, énigmatique, a remplacé celui, plus pragmatique, du monde totalitaire.

\section{Géographie du pouvoir}

7 Dans ce monde où l'autorité principale est celle d'Abi, la société est structurée selon une géographie étrange, que l'on devine centrée autour d'un quartier central, la Cité de Dieu. À partir de ce point, différents quartiers forment des cercles, le plus proche étant le plus privilégié, le plus éloigné étant le plus pauvre et isolé. Cette structure rappelle les géographies dystopiques d'œuvres comme Métropolis, ou Snowpiercer dans lesquelles la basse population vit éloignée de la haute. Dans Métropolis, les riches vivent en hauteur et les pauvres dans les bas-fonds. Dans Snowpiercer, les riches sont dans les wagons de tête et les pauvres en queue. Il est intéressant de souligner que dans 2084, l'organisation géographique que l'on imagine en cercles concentriques, ne souffre la présence d'aucune verticalité si ce n'est la fameuse Cité de Dieu, signe que l'Abistan est une terre où l'individu est effacé dans le collectif, où l'ambition n'a plus lieu d'être et où la soumission passe par une unique structure verticale, qui domine et observe les alentours :

Ati et Koa se sentaient écrasés par la majesté, tout était colossal, démesuré, au-delà des dimensions humaines (...). L'entrée de la Cité était marquée par une voûte cyclopéenne, appelée la Grande Arche du Premier jour, dont l'arc se perdait dans les nuages. Les piliers étaient à l'avenant, ils comptaient soixante siccas de large et trois cents de portée sous voûte, et s'imbriquaient dans le pharaonique rempart qui ceinturait la Cité de Dieu - écrin fabuleux de la Kiiba, de l'Abigouv, de la Grande Mockba (...). Toute la substance du monde était là, concentrée entre ces 
inébranlables remparts : l'éternité, la puissance, la majesté et le mystère. Ailleurs était le monde des hommes, un jour il existerait peut-être (idem : 181). l'Abistan. De fait, la verticalité est depuis toujours un symbole fort du religieux, que ce soit à travers le mythe de Babel ou les utopies architecturales des bâtisseurs de cathédrales. En décrivant ainsi la Cité de Dieu de l'Abistan, Sansal renforce le caractère extrêmement religieux de sa dystopie, mais aussi la fonction totalitaire de cette forme d'architecture. Un point déjà essentiel dans 1984 :

The Ministry of Truth -Minitrue, in Newspeak-was startlingly different from any other object in sight. It was an enormous pyramidal structure of glittering white concrete, soaring up, terrace after terrace, 300 meters into the air. From where Winston stood it was just possible to read, picked out on its white face in elegant lettering, the three slogans of the Party: WAR IS PEACE FREEDOM IS SLAVERY IGNORANCE IS STRENGTH. (Orwell, 1948: 6)

Cette géographie, cette architecture du pouvoir, est plus directe et pernicieuse encore en Abistan, car le monde est si grand que la Cité de Dieu ne peut être vue de loin. A contrario, les bâtiments qui la composent sont le cœur de la surveillance de chacun, à la manière de Barad Dûr dans The Lord of The Rings. La comparaison pourrait paraitre alambiquée, mais il n'en est rien, car il y a chez Sansal une mystique, voire une mythologie, dont l'œil est une donnée fondamentale. Cet œil voit tout, à travers un pouvoir qui ressemble soit à de la superstition ou une métaphore d'un puissant appareil de surveillance. Dans ces quartiers gouvernementaux une des pyramides est décrite en particulier, «sur les quatre faces de son pyramidion, tout là-haut, près du ciel de Yölah, l'œil magique de Bigaye scrutant sans répit le monde et les âmes qui l'habitaient " (Sansal, 2015 : 178). L'œil de Sauron chez Tolkien, celui qui voit tout, est le même que l'œil d'Abi au centre du territoire, maléfique et démiurgique. Réel ou non, il instaure la terreur dans le cœur des habitants de l'Abistan, et le respect teinté de peur, fantasme suprême d'un Machiavel. Le project 1984, concours lancé par des architectes ${ }^{2}$ pour représenter Océania, décrit lui aussi cet état de fait :

Four towers rise above the city like muscular trunks in a grass field. Their scale obliterates any possible question about the intentionality of their disproportionate size. The exaggerated disparity between them and the urban fabric could not have been accidental. The towers were unquestionably built to be the main focus, the sole object of attention. They are by lengths the most important buildings in the city. The towers deliver an explicit message of datum and order. Visible from any point in the city, the towers exploit the potential of architecture as iconography. They are archetypes of power. (Extrait de Project, 1984)

Les quatre tours de 1984 s'opposent donc à cette unique Cité de Dieu de 2084. La différence est de taille, car si quatre tours représentent déjà l'ordre et la puissance, une géographie formée d'un lieu de pouvoir central symbolise un contrôle plus puissant encore. Ce contrôle, c'est celui d'un pouvoir centralisé, d'un œil situé au milieu du territoire, comme si l'Abistan formait une spirale avec en son centre l'œil d'une tempête, plus paisible, plus riche, du moins en apparence. Cette mutation de la géographie de l'Océania de 1984 révèle donc deux éléments primordiaux dans la dystopie voulue par Sansal :

- Le pouvoir est plus centralisé, nous l'avons dit, et l'œil d'Abi a une présence presque physique, plus effrayante encore, dans la Cité de Dieu.

- Privés de la vision de la Cité de Dieu, trop éloignée des territoires les plus pauvres, les habitants de l'Abistan n'ont pas de repère concret du pouvoir et de l'autorité de leur pays, si ce n'est à travers les affiches de propagande et les représentants de la Loi. Ce qui ajoute à l'absurdité d'un monde déjà bien obscur. 
11 Et reste cet aspect féérique ridicule, qui achève le portrait borgésien d'un Abistan chaotique. La Cité de Dieu est, nous l'avons cité plus haut, une cité riche à outrance, comme un pastiche outrancier des Mille et une nuits. Sansal réitère ici l'hypocrisie d'une théocratie dans laquelle les pauvres vivent dans des taudis, et les officiels dans des bâtiments luxueux, à la gloire d'un dieu et d'un prophète qui régissent le monde.

\section{Mutation du langage}

12 À l'instar de 1984, 2084 accorde une place importante au langage. Dans l'Océania d'Orwell, la novlangue de l'Angsoc était un moyen de réduire l'étendue conceptuelle du langage afin d'empêcher à terme le prolétaire de penser par lui-même. Pourtant elle cohabite encore avec le langage anglais normal. En Abistan, le langage a été totalement effacé, et a été remplacé par l'Abilang :

Comme elle inclinait à la poésie et à la rhétorique, elle a été effacée de l'Abistan, on lui a préféré l'Abilang, il force au devoir et à la stricte obéissance. Sa conception s'inspire de la novlangue de l'Angsoc. Lorsque nous occupâmes ce pays, nos dirigeants de l'époque ont découvert que son extraordinaire système politique reposait non pas seulement sur les armes mais sur la puissance phénoménale de sa langue, la novlangue, une langue inventée en laboratoire qui avait le pouvoir d'annihiler chez le locuteur la volonté et la curiosité. (Sansal, 2015 : 260)

Remarquons dans cet extrait que Sansal explique très clairement que l'Abistan est un territoire conquis sur l'ancienne Océania et qu'ils se sont inspirés de la novlangue instaurée par ses anciens dirigeants. On pourrait de fait arguer que Sansal joue avec la géographie d'Orwell et que les conquérants sont arrivés d'Europea. Pour revenir à la question du langage, 2084 présente une mutation forte, puisque le concept de la novlangue est poussé jusqu'au bout de sa logique. L'Abilang est le stade ultime du langage manipulatoire. Dans les dystopies d'Orwell et Sansal, le monde à venir est un monde où la langue même est réduite à l'esclavage et où les masses perdent le contrôle de certaines facultés :

Mais au fond, la question de la vraisemblance scientifique du novlangue n'est qu'accessoire pour l'écrivain. Il a inventé un dispositif extraordinairement efficace pour confronter le lecteur à une réalité autrement plus quotidienne et aliénante, celle de la manipulation des masses par la parole officielle, qu'elle soit de nature doctrinaire ou de nature publicitaire. (Barbaud, 1983 : 72)

14 Comme l'explique Philippe Barbaud, la vraisemblance n'est pas chère à l'écrivain, seul compte la volonté d'enfoncer le lecteur dans un cauchemar plus large encore. Mais cette discussion sur le caractère scientifique d'une "déprogrammation » de l'individu par le langage reste ouverte :

Mais pour le linguiste non générativiste, il se pourrait que le conditionnement linguistique des masses parlantes par un pouvoir quelconque, grâce à l'action convergente des puissants moyens d'intervention directe sur les énoncés qui sont accessibles aux locuteurs, puisse peu à peu modifier leur performance en matière de langage. Le phénotype exerçant une censure de plus en plus soutenue sur le génotype, les mécanismes bien connus d'adaptation, de régulation et de transfert s'enclencheraient irréversiblement jusqu'à ce que soit atteint un nouvel état d'équilibre stable entre génotype et phénotype linguistiques. Il s'ensuivrait une modification profonde de la grammaire universelle découlant de la nouvelle téléonomie de ses éléments constitutifs. Celle-ci deviendrait alors le produit 
robotisé à partir duquel se construiraient, mentalement parlant, des humanoïdes

sachant s'énoncer mais ne sachant plus penser. (idem : 82) totalitarismes. Réduire le langage à l'expression la plus simple, jusqu'à réussir à transformer les locuteurs, non seulement dans leur expression orale, mais aussi dans leur conscience et leur capacité à penser. En Abistan, théocratie cauchemardesque, l'Abilang n'est alors qu'un outil de soumission supplémentaire, un outil de contrôle et un destructeur de concepts, afin qu'il ne reste plus que Yölah et Abi, son Prophète, comme seuls dépositaires de la vérité.

\section{L'Abistan est le prochain stade de l'Océania} futuriste. Il y a, dans 2084, une volonté de rappeler que le politique peut déjà porter le religieux et vice-versa. La mutation d'une dystopie politique (Orwell) à une dystopie religieuse n'est alors que le schéma d'une structure cyclique dans le fonctionnement des sociétés. Les théocraties passent, les démocraties adviennent, et tout recommence ${ }^{3}$. L'Abistan n'est, et c'est l'essentiel, que la suite logique de l'Océania dominée par Big Brother, et Abi est d'ailleurs le remplaçant de cette figure stalinienne, comme la description du portrait le suggère : «(...) ce portrait, il faut le savoir, il était l'identité du pays, il se réduisait en fait à un jeu d'ombres, une sorte de visage en négatif, avec au centre un œil magique pointu comme un diamant, doté d'une conscience capable de perforer des blindages. » (Sansal, 2015 : 29-30) angle. L'Abilang est le prochain stade de l'Océania, un territoire dévasté sur lequel le religieux permet davantage de contrôle encore que ne le pouvait l'Angsoc de 1984, et ce même s'il revêtait déjà un caractère sacré, puisque comme le dit Yvon Desrosiers, «La religion peut donc être vue comme un système de symboles qui s'organisent en récits que sont les mythes et en gestes que sont les rituels " (Desrosiers, 1986). Ces rituels, nous l'avons dit, étaient déjà présents en Océania, à travers « The Two Minutes of Hate » par exemple, et ceux de l'Abistan n'en sont que des reprises où le nom de Yölah est prononcé.

On apprend à travers divers indices que l'Abistan était un des territoires en guerre contre l'Océania, et que ce dernier a fini par céder face à la volonté de conquête du premier : « Il saurait que le seul pays qui avait résisté aux forces de l'Abistan, parce que gouverné par un dictateur fou nommé Big Brother qui avait balancé dans la bataille tout son arsenal nucléaire, était l'Angsoc... ou l'Ansok, mais au final il était tombé et avait été noyé dans son propre sang. » (Sansal, $2015: 240)$

Ou encore : « un cartouche gravé dans la pierre au-dessus du berceau du monumental portail de la forteresse révélait une date, si c'était bien une date, 1984, entre deux signes cabalistiques effrités » (idem : 42). Les signes cabalistiques ne sont là que pour rappeler le point de départ d'une histoire mythique et mystérieuse, puisque les origines d'une dystopie sont, et Orwell l'avait déjà très bien écrit, obscures, réécrites, mystifiées. 2084 n'y échappe pas, et l'histoire du Prophète Abi sera réécrite au gré des découvertes archéologiques gênantes, notamment celle d'un village étrange, qui indique qu'une autre civilisation existait avant celle de l'Abistan (chose impossible) et le gouvernement s'empresse de faire croire que ce village est en fait un village qu'avait connu Abi, et qu'il 
devenait, de fait, sacré: «La nouvelle mouture du Saint Livre devrait intégrer le fait qu'Abi s'était caché plusieurs années dans ce village miraculeux après qu'il avait fui Our, menacé qu'il était par les seigneurs de cette ville corrompue, acquise à Balis et à l'Ennemi. » (idem : 120-121).

Les artifices, les manipulations de ce gouvernement n'ont rien à envier à celui de l'Angsoc d'Orwell, et pour cause: l'Abilang est l'Angsoc, mais dominé par la religion et l'obscurantisme.

\section{Un hommage à Orwell et un avertissement}

21 Gilles Deleuze nous dirait qu'il n'y a pas une vérité et que la littérature possède sa vérité propre, qui est une vérité transcendantale, celle du symbole, à travers les personnages ou le récit. D'où un dépassement de la vérité. Selon lui, dans sa Présentation de Sacher Masoch. La Vénus à la Fourrure, la littérature produit toujours « une sorte de double du monde capable d'en recueillir la violence et l'excès » (Deleuze, 1967: 33). De fait, la dystopie est un réel fabriqué, censé refléter les extrêmes de nos sociétés, et apporter une réflexion profonde qui est cette vérité deleuzienne.

2084 est ainsi, dans son titre, dans ses intentions, et dans son idéologie, la suite de 1984. Il décrit une mutation dystopique inédite dans la fiction, mais se veut surtout un avertissement quant au présent, car comme l'explique Valérie Stienon :

À la fois genre littéraire et échantillon d'un imaginaire social, le récit dystopique est éminemment idéologique. Pourtant, ce sont moins les innovations techniques et politiques elles-mêmes, que les spéculations, les craintes et les tabous dont elles sont porteuses qui font l'objet de ces fictions. Aussi convient-il de ne pas surfaire l'aspect normatif de réussite ou d'échec du monde représenté pour mieux étudier ces productions comme les résultats d'un certain discours social. (Stienon, 2012:2)

2084 est clairement un hommage à 1984 et nous avons pu voir les nombreux échos qu'il y fait. Néanmoins, ces reprises, ces transformations, ne sont pas seulement là pour créer une dystopie de plus. Il y a, chez Sansal, cette volonté d'avertir son époque quant à l'islamisme représenté par Daech certes, mais surtout par des mutations bien plus larges et pernicieuses, ce qu'il explique lui-même dans une interview accordée au Figaro:

Cet « État » sème la terreur et le chaos, mais est appelé à disparaître. En revanche, l'islamisme, dans sa version totalitaire et conquérante, s'inscrit dans un processus lent et complexe. Sa montée en puissance passe par la violence, mais pas seulement. Elle se fait également à travers l'enrichissement des pays musulmans, la création d'une finance islamique, l'investissement dans l'enseignement, les médias ou les activités caritatives. L'Abistan est le résultat de cette stratégie de long terme. (Interview de Boualem Sansal pour Le Figaro, 2015)

L'Abistan est donc le prochain stade de la dystopie, celui qui viendra après l'Angscoc dépeint par Orwell. 2084 est alors davantage qu'une mutation fictionnelle, c'est une mutation idéologique, dans laquelle le retour du religieux menace le monde et présente une forme de totalitarisme :

Oui, c'est le monde que décrit Orwell dans 1984, très proche de celui que nous connaissons aujourd'hui où les individus sont domestiqués par la consommation, par l'argent, mais aussi par le droit. Ce dernier domine désormais les politiques, mais aussi le bon sens populaire. Le but est de conditionner l'individu. Cependant, ce système fondé sur l'alliance entre Wall Street et les élites technocratiques arrive à épuisement en même temps que les ressources naturelles. Dans cinquante ans, il n'y aura plus de pétrole et le problème de la répartition des richesses sera encore 
accru. Il faudra mettre en place un système encore plus coercitif. Une dictature planétaire, non plus laïque mais religieuse, pourrait alors se substituer au système actuel qui devient trop compliqué à cause de la raréfaction des ressources. (Interview au Figaro, 2015)

La conviction profonde de Georges Orwell était que le monde de demain allait être dominé par la surveillance, la restriction des libertés individuelles et le politique totalitaire. Celle de Boualem Sansal est que notre civilisation capitaliste va disparaître et laisser la place au conquérant le plus fort, soit le religieux le plus fanatique. Orwell avait en partie vu juste, l'avenir nous dira si 2084 était le 1984 du siècle à venir.

\section{BIBLIOGRAPHIE}

COHEN, Henri et alii (1983). Orwell a-t-il vu juste? Une analyse sociopsychologique de 1984. Québec :

Presses de l'Université du Québec.

DELEUZE, Gilles (1967). Présentation de Sacher-Masoch : le froid et le cruel... suivi du texte intégral de La Vénus à la fourrure. Paris : Minuit, coll. « Arguments ».

DESROSIERS, Yvon (1986) «La religion dans 1984», in COHEN, H., LÉvy, J.-J. et al., Orwell a-t-il vu juste?: une analyse sociopsychologique de 1984. Montréal : PUQ.

LABROUSSE, Nathalie (2013). Pourquoi des dystopies? Vallet : Éditions M. Editer.

ORWELL, Georges (1948). 1984. ebook en ligne, version originale : $<U R L$ :https://

www.planetebook.com/ebooks/1984.pdf>

ORWELL, Georges (1972). 1984, traduction française par Amélie Audiberti. Paris : Gallimard, coll. Folio.

SANSAL, Boualem (2015). 2084. Paris : Gallimard.

SLAUGHTER A. Richard (2003). Futures Beyond Dystopia. Londres : Routledge.

STIENON, Valérie (2012). « Dystopies de fin du monde, une poétique du désastre », Culture,

Université de Liège.

Projet de WAI THINK TANK sur l'architecture d'Océania dans 1984 :<URL: http://

waithinktank.com/Project-1984>.

Interview de Boualem Sansal accordée au Figaro le 4 septembre $2015:<U R L:$ http://

www.lefigaro.fr/vox/culture/2015/09/04/31006-20150904ARTFIG00401-boualem-sansal-du-

totalitarisme-de-big-brother-a-l-islamisme-radical.php>

\section{NOTES}

1. Georges Orwell, 1984: 14-16.

2. Accessible sur http://waithinktank.com/Project-1984.

3. La Turquie en est un bel exemple sous l'influence d'Erdogan. 


\section{RÉSUMÉS}

Autres temps, autres peurs. Lors de la rédaction de 1984, Georges Orwell voyait se profiler le spectre des totalitarismes après la Seconde Guerre Mondiale. Un futur sombre pour un monde cauchemardesque, dans lequel l'individu est dissout dans la communauté, l'amour est absent, l'obéissance un devoir et la surveillance totale. Si les dystopies qui se sont développées à la suite d'Orwell se sont chacune intéressées à une particularité de l'idéal sociétal (le bonheur, la famille par exemple), 2084 est certainement celle qui se réclame le plus de 1984. Pourtant le monde décrit par Boualem Sansal est en apparence bien différent car théocratique et sans technologie forte. Mon propos sera pourtant de montrer que le roman dystopique de Boualem Sansal est une suite directe de 1984 et que cette mutation de l'univers dystopique de Georges Orwell est un signal d'alarme lancé par Sansal à une Europe en déclin.

Different times, different fears. While writing 1984, Georges Orwell saw the ghosts of totalitarism arise in the wake of WWII. A dark future for a nightmarish world, in which the individual is dissolved in the community, love is absent, obedience is a duty and surveillance is total. If the dystopias following Orwell were each interested in a particular aspect of social ideals (happiness, family, for example), 2084 is certainly 1984's clearest heir. However, the world described by Boualem Sansal is in appearance very different in its theocracy and its lack of technology. Nevertheless, my purpose will be to show that the dystopian novel by Boualem Sansal is a direct sequel of 1984 and that this mutation of Orwell's dystopian universe is an alarm signal to a declining Europe.

INDEX

Mots-clés : Dystopie, Orwell, Sansal, 2084, 1984

Keywords : Dystopia, Orwell, Sansal, 2084, 1984

\section{AUTEUR}

\section{REGIS-PIERRE FIEU}

Université du Québec à Montréal

regispierre.fieu@gmail.com 